\title{
Pavement Crack Image Segmentation Method based on Multiple Scale and Differential Box Dimension
}

\author{
Gao Ting, Liu Wei, Yang Yang and Wang Weixing \\ School of Information Eng., Chang'an University, China \\ Corresponding author: Wang Weixing, Email: wxwang@chd.edu.cn
}

\begin{abstract}
In the process of highway pavement crack detection and recognition, the critical step is to segment the pavement crack images, in order to obtain accurate feature information of extracted pavement cracks. This paper presents a new method. Firstly, it Shrink the image by analyzing the crack direction, both effectively removing noise and keeping the target information, and then use the logarithmic transform for image enhancement, and subsequently apply for the improved differential box-counting method for image segmentation. In experiments, a number of crack images from an online acquisition system were tested by using our Windows program, compared to other traditional methods, the new method can effectively extract the pavement cracks, and is good for anti-noise performance.
\end{abstract}

Keywords: pavement crack, image segmentation, image shrink, differential box-counting dimension

\section{Introduction}

In modern society, highway especially the freeway plays an important role. Due to a large number of vehicles using the road every day, over time leading is to damage to the road pavement. Without an appropriate maintenance measure, it will reduce the quality of the road surface and be a direct threat to road safety, therefore, a lot of researchers have started to do this kind of studies [1-2].

The most common types of road damages are cracks which can be divided into different types. A crack usually presents to be thin, continuous and dark, and can be detected from the road surface texture. The most common disaster and often the initial worsening sign on the surface of the highway appear first as a single crack because of prolonged using the road, more staggered single cracks will come into being a network of cracks [2-4]. Therefore, when the road surface emerges a single crack, the timely detection and maintenance is so essential to avoid greater losses.

Crack detection is a very critical task in the road network maintenance. In the past few decades, the road damage was inspected by artificial vision, which represents a task of very expensive and time-consuming and financial resources. Since the manual testing method is time-consuming, and affected by the subjective judgment of the testing personnel, different persons give out different judgment results, the test results are usually not so accurate [3-5]. However the digital image processing technology is emerging and thriving, and is widely used in various fields, which offers the possibility of automatic pavement crack detection and recognition.

To overcome manual detection disadvantages, many scholars in the world have been doing research and testing on the task of automatically crack detection and recognition [6-7]. In 2006, Alekseychuk [6] proposed a crack target detection algorithm based on the dynamic optimization, which is on the base of graph theory analysis, in which the cracks were seen as path lines with nodes having similar characteristics. In 2009, Katakam from University of Toledo [8] divided an image into blocks, used local thresholds for each 
sub-block segmentation processing, whereby the information of cracks can be obtained; $\mathrm{Chu}$, etc. [9] separated a damaged road image into blocks, adopted the gray scale variance of each sub-block image, and mean and minimum gray scale variance values of all sub-blocks to describe the texture of the image, and then extracted the cracks through artificial neural network; Wang [10-11] suggested an asphalt pavement crack detection algorithm based on morphology and local thresholding; Li, etc. [12-13] raised a pavement crack detection algorithm based on minimum-cost path ,which is based on graph theory analysis. As the textures of the cracks and the road background are the similar, the gray scale contrast is relatively low; the intensities of each point on a crack in the image are inconsistent due to the uneven illumination. Currently automatic pavement crack detection is still a challenging task.

Based on the analysis of the crack detection from the existing research results, this paper proposes a new method, at first it uses the vertical and horizontal projection curves of the gray scales in the image to analyze the type of the cracks, and then shrinks the image according to the type of the cracks (thick or thin) in order to reduce noise while preserving the target feature edges, subsequently improves the detection rate, and finally uses differential box-counting method on fractal theory for image segmentation.

\section{Crack Image Evaluation and Enhancement}

\subsection{Projection Analysis}

There are lots of particular characteristics with the road pavement crack images, such as: (1) relative to the background, cracks are the darker objects, so that the pixels belonging to the cracks should be the local minimum values (i.e., a pixel with the minimum gray value in its local area); (2) having linear characteristic or directivity, the crack is a connected subset of the image, so there is a particular direction. Therefore, according to the typical characteristics of cracks, the project in the vertical direction and the horizontal direction are respectively for the grayscale crack image, namely add up for each column or row of gray values, the formulas are as follows:

$$
\begin{aligned}
& H=\sum_{j=1}^{n} G(i, j) \\
& V=\sum_{i=1}^{m} G(i, j)
\end{aligned}
$$

For the horizontal cracks, the vertical projection curve is flat, while the horizontal projection curve has a significant mutation. For the vertical cracks, the horizontal projection curve is gently undulating, while the vertical projection curve has a larger mutation. For the network of cracks or the oblique crack, the gray scale horizontal projection and vertical projection curves are relatively flat; there are no significant fluctuations or larger mutations with them. According to the distribution of the projection curves, the type of the crack can be determined, which is very useful information for image preprocessing and segmentation, even for crack analysis.

\subsection{Image Shrink}

Usually when obtaining pavement images, a high-precision camera is used; the images obtained are of resolution up to $2048 \times 2048$ pixels, and even up to $4096 \times 4096$ pixels. In experiments on processing the pavement images, an operator must do the process and analysis for each image for image evaluation, if the image size is too large, the computer 
processing speed is slow, which cannot achieve the real-time effect. Hence, before the image processing, the image size is reduced first, for which it should guarantee the image quality, and reduce number of the unnecessary pixels, so that it can improve the efficiency of the image processing.

When capturing an image, inevitably there will be some road disturbances such as shadows, possible debris, and uneven illumination that will make image quality worse. If we directly do image enhancement processing, the image noise can be reduced, which directly affect the subsequent crack identification. Here, first we shrink the image using the following formula:

$\left[\begin{array}{lll}x_{1} & y_{1} & 1\end{array}\right]=\left[\begin{array}{lll}x_{0} & y_{0} & 1\end{array}\right]\left[\begin{array}{ccc}\boldsymbol{S}_{x} & 0 & 0 \\ 0 & \boldsymbol{S}_{y} & 0 \\ 0 & 0 & 1\end{array}\right]=\left[\begin{array}{llll}x_{0} * \boldsymbol{S}_{x} & y_{0} * \boldsymbol{S}_{y} & 1\end{array}\right]$

For the image shrinking, we take appropriate $\boldsymbol{S}_{x}$ and $\boldsymbol{S}_{y}$ values depending on the type of the crack (horizontal or vertical), both to maintain the target information, and to reduce noise.

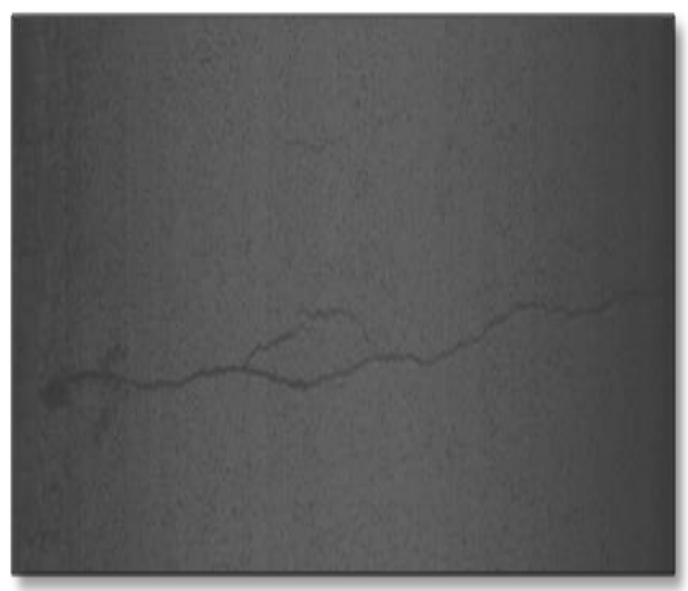

(a) Original image

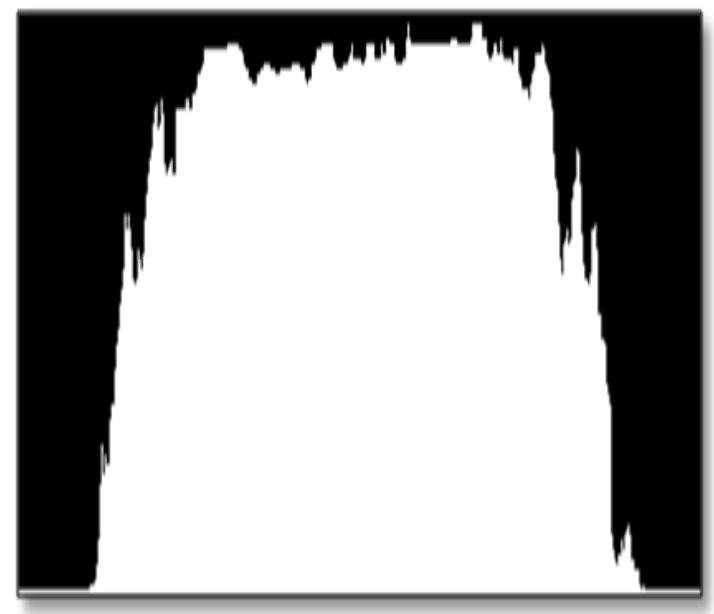

(c) Gray scale projection on vertical

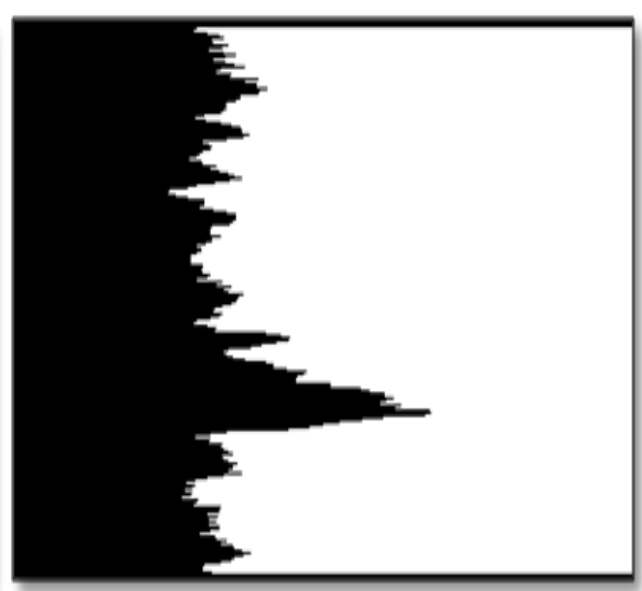

(b) Gray scale projection on horizontal

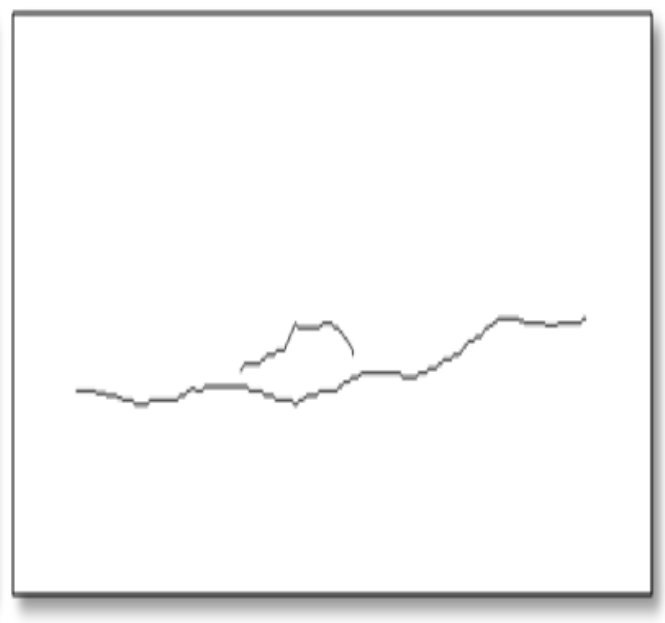

(d) Detection result

Figure 1. Example of Image Projections Corresponding to Cracks, and Crack Detection Result 
One example is shown in Fig.1, the original image is of resolution 4096x2048 pixels, the contrast is very low, the illumination is uneven, and it includes one long horizontal crack. To reduce computing burden we shrink the image 8 times $(512 \times 256$ pixels). For each time of shrinking, we take the minimum pixel gray value of four neighboring pixel values (in shrinking image) as the pixel value in the corresponding position of the new image.

As shown in Fig.1(b), the horizontal gray scale projection histogram has a large valley (see black triangle), which means that there is at least one horizontal crack in the image, before crack detection, on the horizontal direction, the image can be shrunk more times than that on the vertical direction. In Fig.1(c), the right and left parts are darker, that means that the illumination in the image is uneven, two edge parts are darker, if necessary, we may need to enhance image locally not globally. When the information is used for image enhancement and image crack detection, and the detection result can be satisfactory as show in Fig.1(d).

\subsection{Image Enhancement}

For the pavement cracks, the pixels in cracks are darker, however, when the gray scales of the background and objects are the similar to each other, if using a linear transform for image enhancement, it not only enhances the targets, and also increases the same magnitude to the background, so this paper uses a logarithmic transformation to process the gray scale crack images, and the formula is as follow:

$$
t=c \cdot \log _{v+1}(1+v \cdot r), r \in[0,1]
$$

In a crack image, the larger, the base of the logarithmic transformation, the stronger, the emphasis on low gray scale part, so the stronger, the compression of the high gray scale part. For a pavement image, the crack targets are of the lower gray values, while the background pixels are of the higher gray scale values, so when adopting the logarithmic transformation, it is able to expand the target portion with low gray scales, and to compress the background section with high gray scales, which can achieve the enhancement of the low gradation portion. After processing the gray scale pavement images by the logarithmic transform, the gray scale contrast of the crack area with low gray scale values is increased, and the details in darker parts are enhanced.

If the image illumination is uneven too much, as shown in Fig.1(c), the image can be split into three parts, and the parts are enhanced respectively.

\section{Image Segmentation based on Fractal Theory}

In 1973, Mandelbrot first proposed the fractal dimension and fractal geometry idea, and the fractal geometry mainly express the irregular, self-similarity and self-affinity objects in nature by the way of a quantitative description. Compared with the conventional geometrical, the fractal geometry has such features: (1) overall every part of the fractal geometry graph is irregular; (2) on different scales, the systematicness of the pattern is the same [14].

\subsection{Basic Definition and DBC Estimation}

Fractal dimension (FD ) can be used to describe the irregular extent of natural phenomena, and it is an important feature and a fractal measure. Since the fractal dimension combines the spatial information and gray scale information of an image simply and cleverly, it can be a stable characteristic quantity to describe a natural object. There are many definitions for fractal dimension, the basic approach to estimate FD is 
based on the concept of self-similarity. The definition of self-similarity dimension is: for bounded set $F$ in an N-dimensional space, if $F$ can be expressed as the union set of N non-overlapping subsets, $F$ is self-similar. Then the fractal dimension of $F$ is:

$$
D=\lim _{r \rightarrow 0} \frac{\log \left(N_{\varepsilon}\right)}{\log (1 / \varepsilon)}
$$

$N_{\varepsilon}(F)$ is a scale factor. The definition of box dimension (also called entropy dimension, capacity dimension or logarithmic dimension) is: $N_{\varepsilon}(F)$ represents the minimum number of boxes using the sets with the diameter less than $\varepsilon$ to

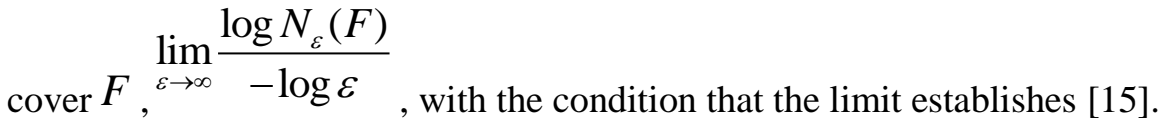

Fractal dimension defined by self-similarity can be understood easily in theory, but it is very difficult to achieve in practical calculations. A common estimation method of fractal dimension is that using box dimension, Sarkar and Chaudhuri proposed an efficient method to calculate fractal dimension based on $\varepsilon_{\text {- covering thought, called differential }}$ box - counting (DBC) method [16], which has better accuracy than the traditional box dimension methods and is more simply to calculate. The following is the basic idea of this method:

The image with the size of $M \times M$ is regarded as a cured surface $(x, y, z)$ in three-dimension space, coordinates $(x, y)$ represent the two-dimension coordinate plane, $z$ represents the gray scale that the point $(x, y)$ corresponding to. The $(x, y)$ plane is divided into small grids of $s \times s$, where $s$ is an integer, $M / 2 \geq s>1$, then $r=s / M$. Therefore in the three-dimensional space, each grid is piled with a lot of small boxes whose volume is $s \times s \times h$, where $h$ represents the height of each single grid. If the gray scale number of the image is $G$, the value of h meets $G / h=M / s$. Assume that the maximum and minimum gray scales of the pixels in the $(i, j)$ grid respectively fall on the boxes of the serial numbers $k$ and $l$. Then the total box number $n_{r}(i, j)$ contained in the grid is:

$$
n_{r}(i, j)=k-l+1=\left[I_{\max }(i, j)-I_{\min }(i, j)\right] / h+1
$$

Where, $I_{\max }(i, j)$ and $I_{\min }(i, j)$ correspond to the maximum and minimum gray scales of the pixels in $(i, j)$ grid. The $n_{r}(i, j)$ of all grids are added up, then the total box number $N_{r}$ covering the image area is

$$
N_{r}=\sum_{i, j} n_{r}(i, j)
$$

The change of $s$ leads to that of $r$, fitting the line $\log \left(N_{r}\right) \sim \log (1 / r)$ by the least square method can calculate the slope, being the fractal dimension $D$. 


\subsection{Improved DBC method}

When calculating the differential box-counting dimension, the scale $r$ is usually selected as a power of 2 , such as $2,4,8,16$ and so on. When $r=2$, the outcome value represents the fractal dimension of $2 \times 2$ block, and so on, when $r$ changes. For pavement cracks, since cracks are of usually relatively thin and dark pixels, if the selected scale $r$ is too large, the calculated local FD represents a sub-block, especially if cracks are minor, even it needs to calculate the fractal dimension of a single pixel to describe the crack.

The smaller the scale $r$ is, the more the local FD can reflect the image details. But when $r=1$, using the traditional box-counting method cannot calculate the fractal dimension. Aiming at this problem, then the method for calculating the fractal dimension is improved.

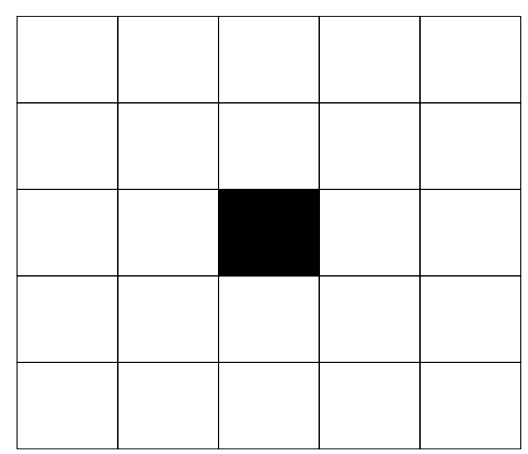

(a) The center pixel in $5 \times 5$ kernel

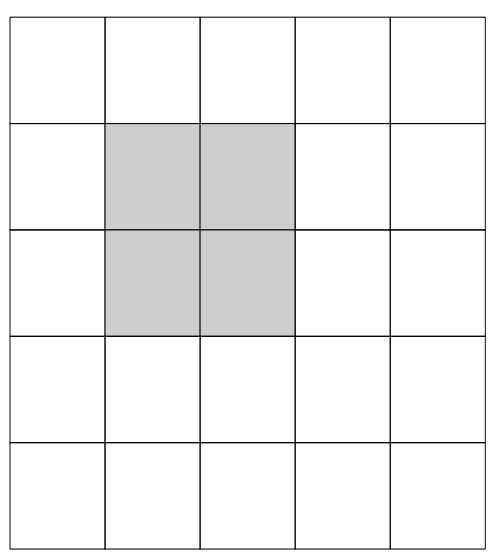

(b) The upper left block in $5 \times 5$ kernel

Figure 2. Center Pixel and its Upper Left Block in 5x5 Kernel

Figure 21(a) shows that, when calculating the box dimension of the current detecting pixel, we calculate the DBC dimension values of its four neighbor $2 \times 2$ blocks, compute its upper left, upper right, lower left and lower right of the four neighborhood blocks, and take the average value of these four values as the fractal dimension of the center pixel.

After above calculations, we do a special treatment for the pixels of the image boundary (a rectangle has four edges). For the four vertices of a rectangle image, we calculate the DBC dimension of the $2 \times 2$ block, which is regarded as the fractal dimension of the vertex pixels; for the other pixels of the image boundary, we compute the two box dimension values of its adjacent left and right blocks, or the top and bottom $2 \times 2$ blocks, take the average value of the two box dimension values as the DBC dimension of the current edge pixel.

\section{Experimental Results and Analysis}

This paper presents a new segmentation method to extract pavement cracks, first it calculates the gray scale vertical and horizontal projection curves, judge type of the crack (horizontal or vertical), then shrinks the image according to the crack type, which can effectively reduce noise and retain the target information, and subsequently uses the logarithmic transform for image enhancement, and finally applies the modified differential box-counting algorithm to detect the crack. The flowchart of this method is as follows: 
In real application, to evaluate road quality, a truck moves on a road, the camera under the truck body takes video film on road surface, therefore a huge number of pavement images are taken in order, for the road within a certain distance. After image acquisition, each of the images (in order) is checked if it involves cracks or not. In addition, the direction of the cracks needs to know if it (or they) diagonal, vertical or horizontal. Normally the crack detection is by manual, which is time tedious and slow process, and the different persons give different evaluation results. Hence we develop a vision system to automatically and quickly detect cracks.

The raw image size selected for experiments is $4096 \times 2048$ pixels, in which each pixel is 8-bit 256 gray scales. A number of pavement images were selected. For the images of concrete pavement, the logarithm transform can be ignored as the contract of the crack and the pavement background is obvious. In the following, three crack images are presented, and they have diagonal, vertical and horizontal cracks respectively.

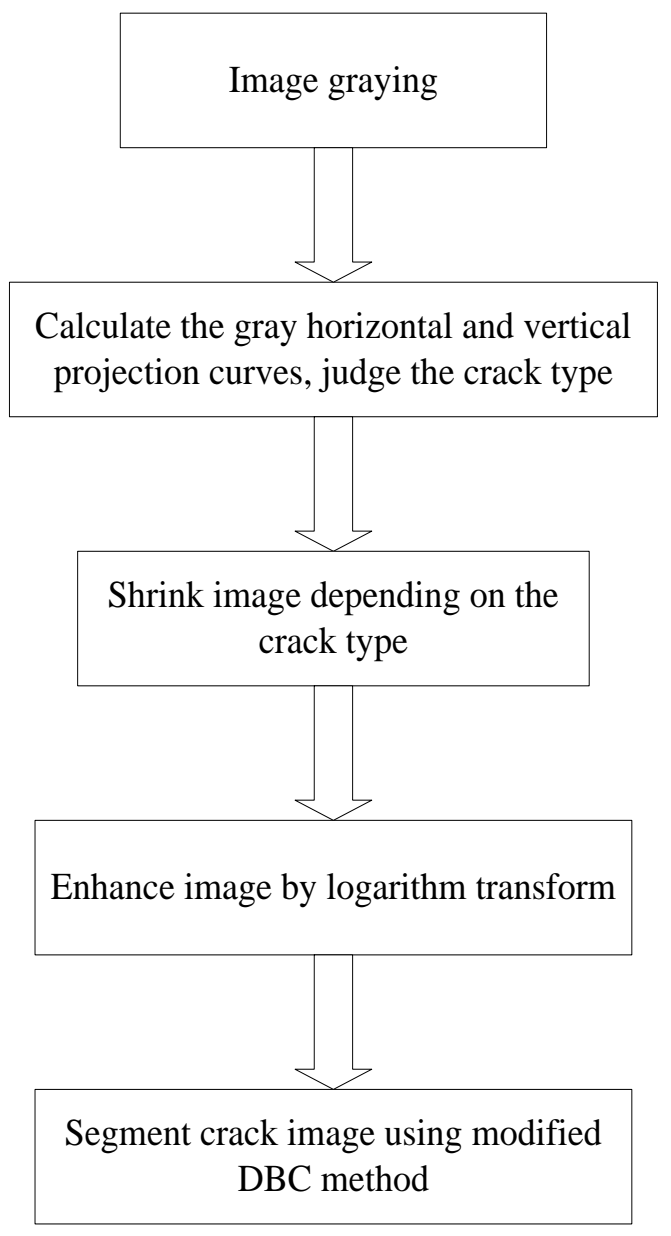

Figure 3. Flowchart

To develop an automatic crack detection system, we made a Windows program including a number of functions, the program layout is illustrated as in Fig.4. More than 200 pavement crack images are tested, the program works satisfactory. In the following, three typical images and their segmentation results comparing to other algorithms are described.

In Fig.5(a), the contrast between crack and background is high, illumination is even, the image should be good by using a simple thresholding algorithm for image segmentation, but the background includes a lot of dark and white spot noise, which 
affects image segmentation result. As tested, the simple Canny edge detector (discontinuous based algorithm) or Otsu thresholding algorithm (grey scale similarity based algorithm) cannot perform image segmentation well, the Canny edge detection gives out some spurious edges and noise, and Otsu thresholding results in too many noise, as shown in Fig.5(b, c). The new method can make a satisfactory image segmentation result (Fig.5(d)), where, the crack is clearly extracted in addition to having a few noise spots. For the small spot noise, a simple object area threshold can be applied to remove them.

The image in Fig.6(a) has a vertical crack, the contrast between the crack and background is low, the up part is darker than down part, the illumination is uneven. Compared to Fig.5, the main difference is that when using Otsu thresholding, the up part includes more noise. The new algorithm result is still satisfactory. Of course, some other intelligent algorithms can also be used for such an image, but their working speed might be not suitable.

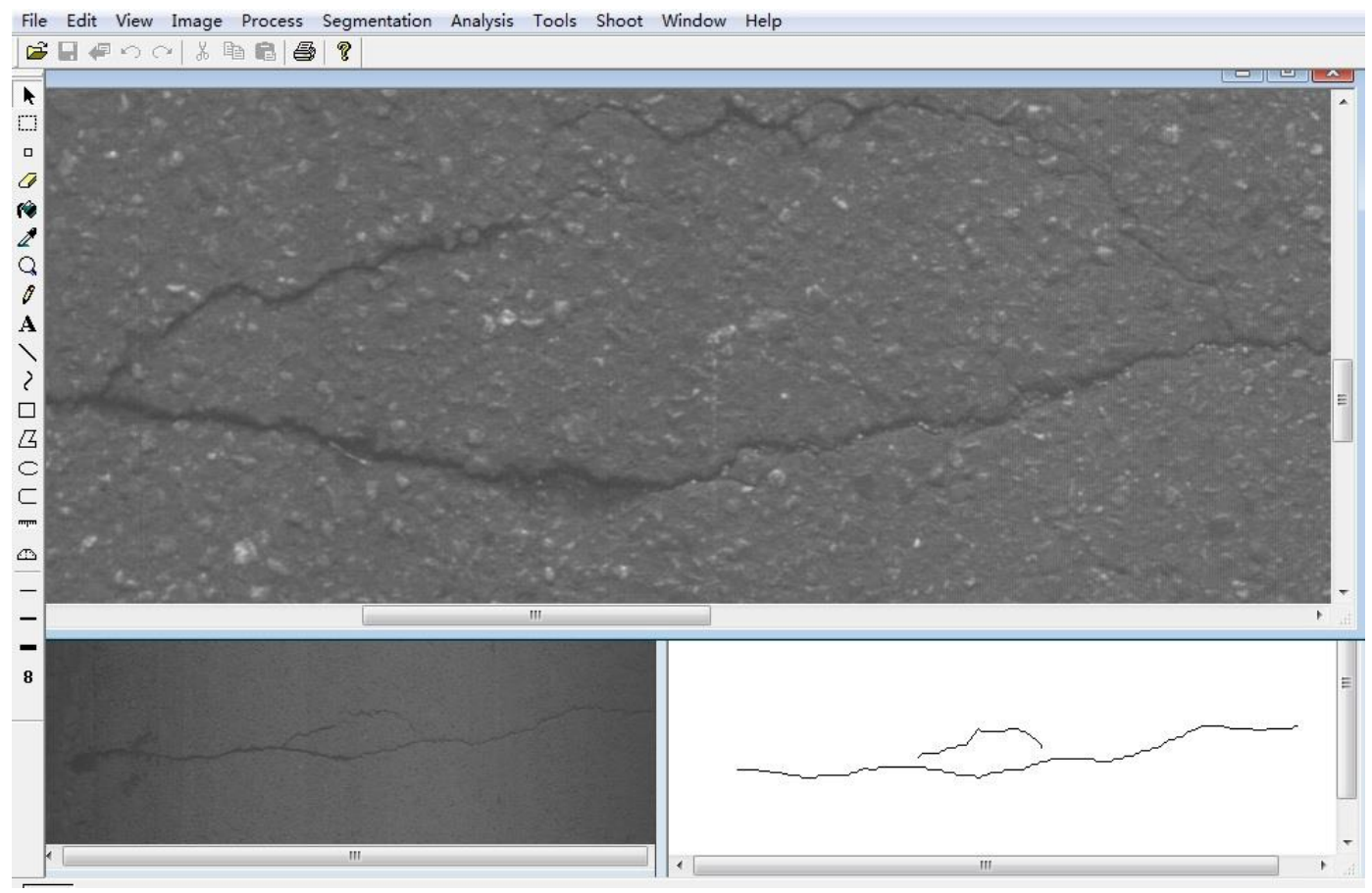

Figure 4. Windows Program Layout for Pavement Crack Detection: Original Image (at upper) Resolution is 4096x2048 Pixels, it is Shrank 8 Times (left-bottom), and the Detection Result is Shown on Right-bottom

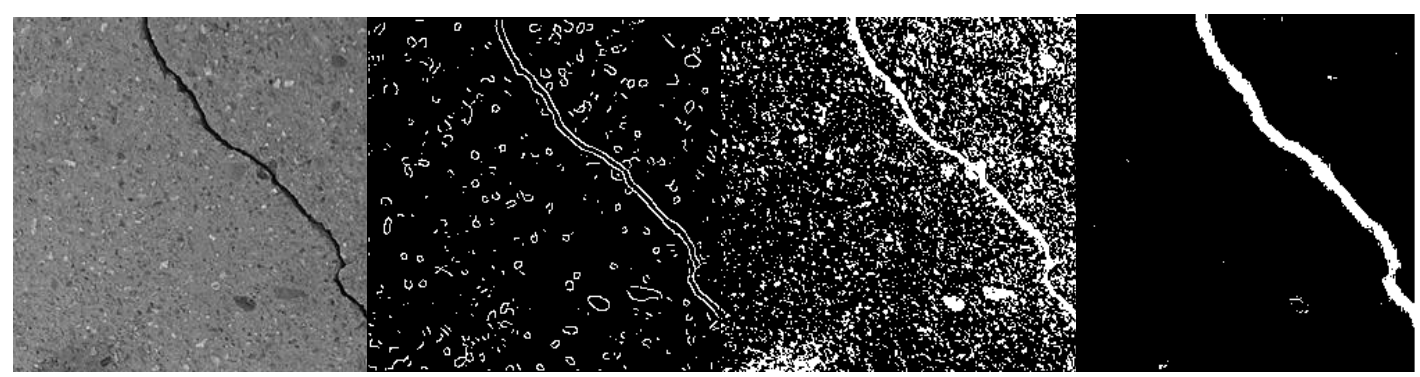

(a) Origina image

(b) Canny detection (e) Otsu thresholding

(f) New method

Figure 5. Diagonal Crack Detection Results 


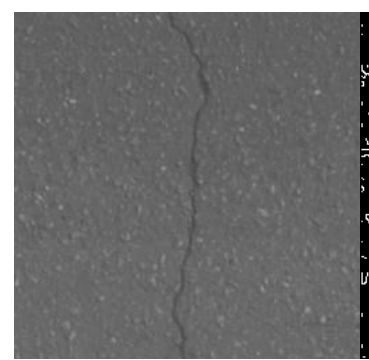

(a) Origina image

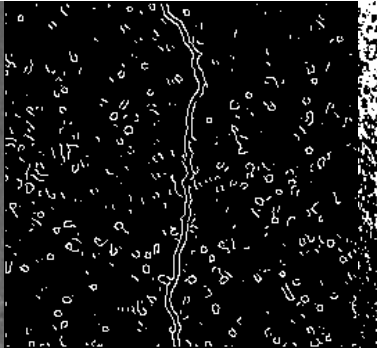

(b) Canny detection

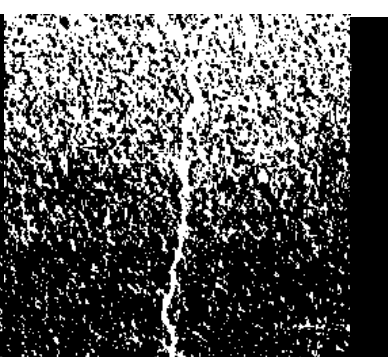

(e) Otsu thresholding

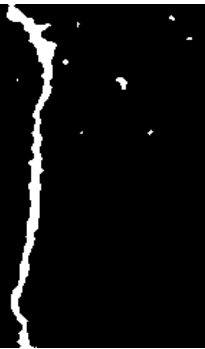

(f) New method

Figure 6. Verticle Crack Detection Results

The image in Fig.7(a) has a horizontal crack, the contrast between the crack and background is low, the right part is darker than the left part, the illumination is uneven. Compared to Fig.6, the main difference is that when using Canny edge detector and Otsu thresholding algorithm, the right part always have more noise, but the new algorithm performs well too.

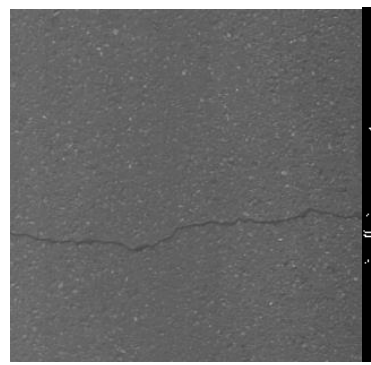

(a) Origina image

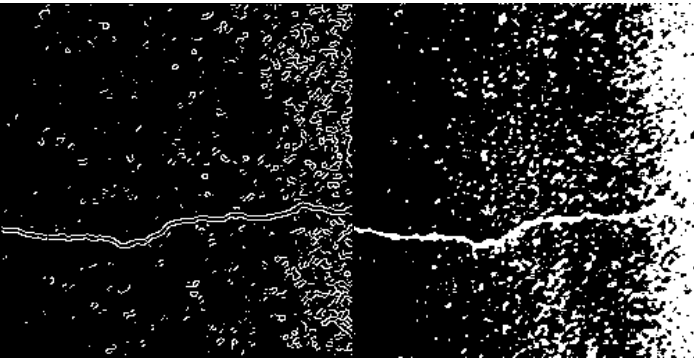

(b) Canny detection

(e) Otsu thresholding

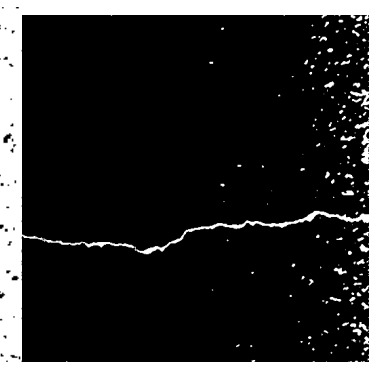

(f) New method

\section{Figure 7. Horizontal Crack Detection Results}

The above test results show that since the asphalt pavement is rough, pavement material itself causes a lot of isolate-distributed noise points, and the gray scales of dark noise pixels are close to that of the crack pixels. Otsu thresholding and Canny edge detection methods [17-18] are sensitive to noise, the results contain different noise which affect crack identification; the new method can extract cracks from background well, for which the pavement crack is extracted more accurately, and there is only a small amount of noise pixels in the crack image.

\section{Conclusion}

In this paper, a pavement crack segmentation method based on the modified DBC algorithm (fractal analysis) has been presented. It first evaluate if an input image has cracks, which direction the cracks are in, then based on the evaluation, the image is shrunk, into accepted minimum size, subsequently the shrunk image is enhanced and segmented. The whole procedure is coded into a Windows program with $\mathrm{VC}++.200$ hundred images are tested, and it works well. The test proves that this method performs better than other algorithms (traditional discontinuous based and similarity based algorithms), and it can make the crack location more accurate, better anti-noise performance, and most isolated noise point removal. The new method provides a new effective way for pavement crack detection. 


\section{Acknowledgements}

This research is financially supported by the National Natural Science Fund in China (grant no. 61170147).

\section{References}

[1] Sindhu Ghanta, Ralf Birken and Jennifer Dy. Automatic road surface defect detection from grayscale images [C]. Society of Photo-Optical Instrumentation Engineers (SPIE) Proceedings, (2012), 8347.

[2] Wang Weixing, Wang Fengping, Huang Xiaojun, Song Junfang. Rock fracture image acquisition using two kinds of lighting and fusion on a wavelet transform, Bulletin of Engineering Geology and the Environment, Online ISSN 1435-9537, DOI 10.1007/s10064-015-0747-4, (2015), 05, Publisher: Springer Berlin Heidelberg.

[3] Chang-Xia Ma, Chun-Xia Zhao, Ying-kun Hou. Pavement Distress Detection Based on Nonsubsampled Contourlet Transform[J]. IEEE. (2008): 28-31.

[4] Hoang-Nam Nguyen, Tai-Yan Kam, Pi-Ying Cheng. An Automatic Approach for Accurate Edge Detection of Concrete Crack Utilizing 2D Geometric Features of Crack [J]. Journal of Signal Processing Systems, (2014), 77 (3): 221-240.

[5] Wang Weixing and Chen Liangqin, (2015), Flotation bubble tracing based on Harris corner detection and local gray value minima, Minerals, (2015), 5(2), 142-163, doi: 10.3390/min5020142.

[6] Alekseychuk O. Detection of crack-like indications in digital radiography by global optimization of a probabilistic estimation function. Ph.D. thesis, BAM-Dissertationsreihe, 2006, Band 18, Berlin.

[7] W X Wang, W S Li and X Yu, (2012), Fractional differential algorithms for rock fracture images, The Imaging Science Journal, Vol.60, IMAG 93 @ RPS 2012,pp.103-111.

[8] Katakam N. Pavement crack detection system through localized thresholding[D]. The University of Toledo, Deeember (2009).

[9] Chu Xiumin, WANG Rongben, CHU Jiangwei, etc. Study of asphalt pavement surface distress image segmentation[J]. China J ournal of Highway and Transport, (2003), 16(3): 11-14.

[10] Weixing Wang, Linchun Wu. Extraction of Pavement Cracks Based on Valley Edge Detection of Fractional Integral $[\mathrm{J}]$. Journal of South China University of Technology (Natural Science Edition), (2014), 42 (1): 117-122.

[11] Shahid Kabir, Patrice Rivard, Dong-Chen He, Philippe Thivierge. Damage Assessment for Concrete Structure Using Image Processing Techniques on Acoustic Borehole Imagery[J]. Construction and Building Materials, (2009), 23: 3166-3174.

[12] Li Qingquan, Liu Xianglong. Novel Approach to Pavement Image Segmentation Based on Neighoring Difference Histogram Method[J]. IEEE. (2008): 792-796.

[13] Weixing Wang and Sheng Liu, (2015), Online burning material pile detection on color clustering and quaternion based edge detection in boiler, KSII Transactions on Internet and Information Systems, Vol. 9, No. 1, 190-207.

[14] Mandelbrot B B. Fractal: Form, Chance and Dimension[J]. San Francisco: Freeman. (1977).

[15] Kenneth Falcone. Fractal geometry - mathematical foundations and its application [M]. Tseng Wen-song translation. Beijing: People's Posts and Telecommunications Press, (2007): 10-11.

[16] Sarkar N, Chaudhuri B B. An efficient approach to estimate fractal dimension of textural images[J]. Pattern recognition, (1992), 25(9): 1035-1041.

[17] Weixing Wang, 2011, Colony image acquisition system and segmentation algorithms, Optical Engineering, 50(12), 123001 Nov 29, (2011) (SCI: 865CX); doi:10.1117/1.3662398.

[18] Wang Weixing, Zhang Xin, Cao Ting, Tian Liping, Liu Sheng, Wang Zhiwei, (2014), Fuzzy and Touching Cell Extraction on Modified Graph MST and Skeleton Distance Mapping Histogram, Journal of Medical Imaging and Health Informatics, Vol. 4, No. 3, 2014, 2156-7018/2014/4/001/008, doi:10.1166/jmihi.(2014).1264. 